\title{
Metabolomics-Based Studies Assessing Exercise- Induced Alterations of the Human Metabolome: A Systematic Review
}

\author{
Camila A. Sakaguchi ${ }^{1, *}{ }^{\oplus}$, David C. Nieman ${ }^{2}\left(\mathbb{D}\right.$, Etore F. Signini ${ }^{1}$, Raphael M. Abreu ${ }^{1}$ and \\ Aparecida M. Catai ${ }^{1}$ \\ 1 Physical Therapy Department, Federal University of São Carlos, São Carlos, SP 13565-905, Brazil \\ 2 North Carolina Research Campus, Appalachian State University, Kannapolis, NC 28081, USA \\ * Correspondence: sakaguchicamila@estudante.ufscar.br; Tel.: +55-16-3351-8705
}

Received: 10 July 2019; Accepted: 31 July 2019; Published: 9 August 2019

\begin{abstract}
This systematic review provides a qualitative appraisal of 24 high-quality metabolomicsbased studies published over the past decade exploring exercise-induced alterations of the human metabolome. Of these papers, $63 \%$ focused on acute metabolite changes following intense and prolonged exercise. The best studies utilized liquid chromatography mass spectrometry (LC-MS/MS) analytical platforms with large chemical standard libraries and strong, multivariate bioinformatics support. These studies reported large-fold changes in diverse lipid-related metabolites, with more than 100 increasing two-fold or greater within a few hours post-exercise. Metabolite shifts, even after strenuous exercise, typically return to near pre-exercise levels after one day of recovery. Few studies investigated metabolite changes following acute exercise bouts of shorter durations ( $<60 \mathrm{~min})$ and workload volumes. Plasma metabolite shifts in these types of studies are modest in comparison. More cross-sectional and exercise training studies are needed to improve scientific understanding of the human system's response to varying, chronic exercise workloads. The findings derived from this review provide direction for future investigations focused on the body's metabolome response to exercise.
\end{abstract}

Keywords: exercise; sports; metabolomics; metabolism

\section{Introduction}

Acute and chronic physical activity causes extensive adaptations in organs and systems, leading to health benefits [1]. Improvements in technology have allowed investigators to quantify these adaptations using a biological systems approach, overlaying gene information with transcriptomics, proteomics, and metabolomics [1-10]. Combined data from multi-omics approaches will improve scientific understanding regarding the complex modulating effect that physical activity has on the phenotype at the individual level and related molecular mechanisms.

Metabolomics is defined as the simultaneous measurement of numerous low molecular metabolites that participate as substrates, reactants, signaling agents, intermediates, and products of enzyme-mediated reactions [3,4]. Metabolites are the final endpoints of upstream biochemical processes, and closely reflect the expressed phenotype. With the support of advanced analytical platforms and bioinformatics, metabolomics data can provide valuable insights regarding the biological impact of physical activity, pharmacological treatment, nutritional interventions, and other exposures [3].

Global metabolomics procedures were first performed in the 1960s and 1970s when gas chromatography mass spectrometry (GC-MS) was used to measure human metabolites in blood and urine samples [4]. Despite this, metabolomics was considered an emerging field of scientific 
endeavor as late as 2010, the year when the earliest studies investigating exercise effects in human athletes were published [3]. Since then, a growing number of research groups have used metabolomics in exercise-based studies. This is due, in large part, to the widespread availability of mass spectrometry platforms, freely accessible online databases of metabolites such as the Human Metabolome Database (HMDB), the expansion of chemical standards libraries, and advanced bioinformatics support to analyze and make sense of the large volumes of data. The net effect has been an improved capacity to accurately detect a greater number of metabolites and then interpret the overall effect on the human metabolome in a wide variety of matrixes.

This systematic review provides a qualitative appraisal of metabolomics-based studies published during the past decade exploring exercise-induced alterations on the human metabolome. The conclusions derived from this review will provide an evidence-based framework for future investigations.

\section{Results}

A total of 1355 articles were retrieved for this analysis. Of these, 1314 were excluded for not meeting analysis criteria after review of the abstracts. Of the 41 studies selected for full text examination, six were excluded for not meeting analysis criteria. Of the 35 studies included for scoring, 24 achieved a minimum score of 6, and were included in the final analysis (Table 1 and Figure 1).

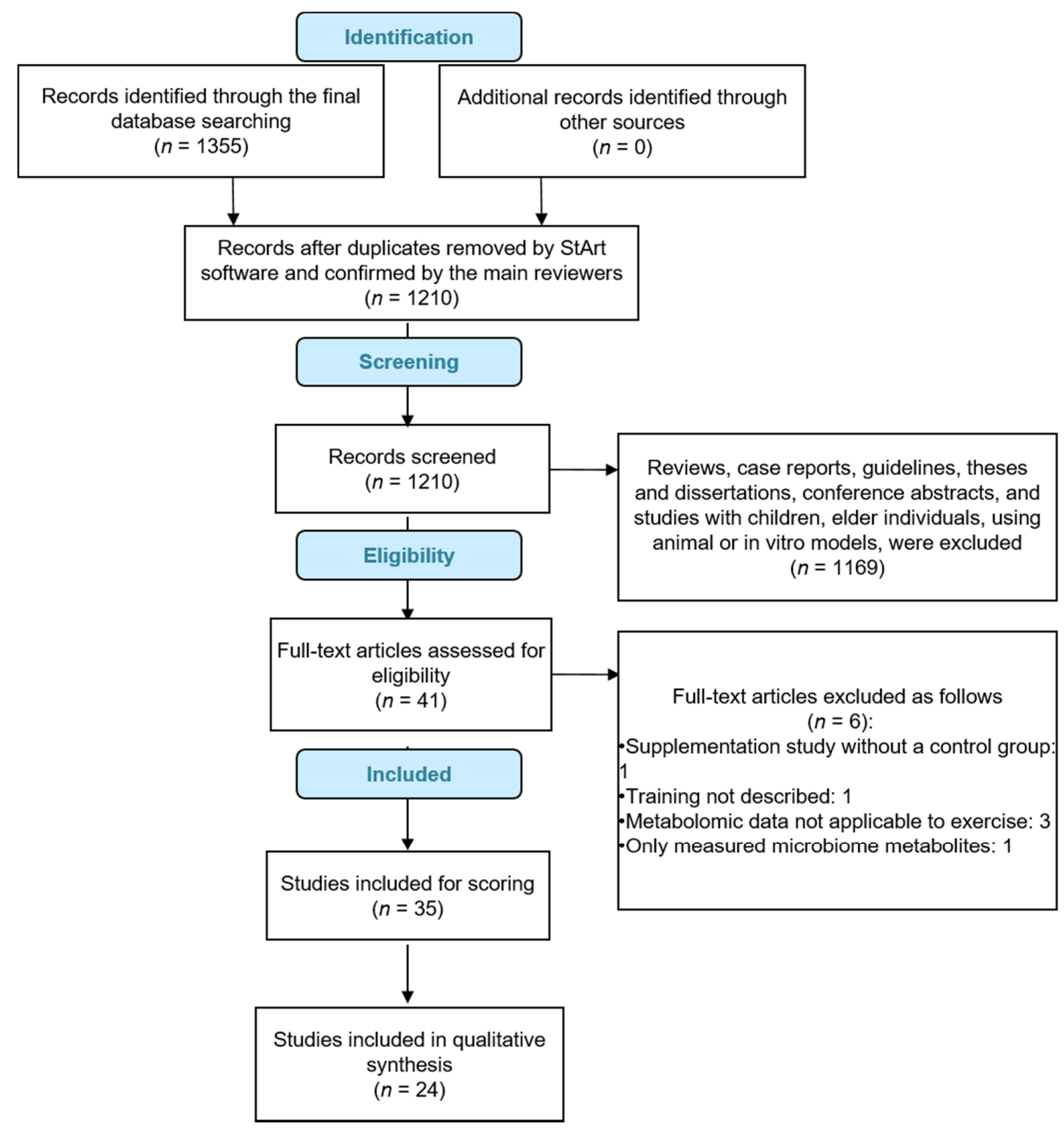

Figure 1. Outcomes of review flow diagram. 
Table 1. Studies classification according to score system.

\begin{tabular}{|c|c|c|c|c|c|c|c|}
\hline \multirow{2}{*}{$\begin{array}{l}\text { Investigators, } \\
\text { Year Published }\end{array}$} & \multicolumn{2}{|c|}{ Research Design } & \multicolumn{2}{|c|}{ Methodology } & \multirow{2}{*}{ Novelty } & \multirow{2}{*}{ Final Score } & \multirow{2}{*}{ Classificatior } \\
\hline & Subjects Number & Studies Characteristics & Analysis Methods & Statistical Support & & & \\
\hline Nieman et al., 2015 [5] & 2 & 2 & 3 & 2 & 2 & 11 & Excellent \\
\hline Jacobs et al., 2014 [6] & 2 & 2 & 3 & 2 & 2 & 11 & Excellent \\
\hline Nieman et al., 2014 [7] & 2 & 2 & 3 & 1 & 2 & 10 & Excellent \\
\hline Nieman et al., 2017 [8] & 2 & 1 & 3 & 2 & 2 & 10 & Excellent \\
\hline Davison et al., 2018 [9] & 2 & 2 & 3 & 1 & 1 & 9 & Excellent \\
\hline Hodgson et al., 2012 [10] & 0 & 2 & 3 & 2 & 2 & 9 & Excellent \\
\hline Karl et al., 2017 [11] & 0 & 1 & 3 & 2 & 2 & 8 & Good \\
\hline Lehman et al., 2010 [12] & 0 & 1 & 3 & 2 & 2 & 8 & Good \\
\hline Nieman et al., 2013 [14] & 0 & 2 & 3 & 1 & 2 & 8 & Good \\
\hline Al-Khelaifi et al., 2018 [15] & 2 & 0 & 3 & 2 & 1 & 8 & Good \\
\hline Knab at al., 2013 [16] & 0 & 2 & 1 & 2 & 2 & 7 & Good \\
\hline Manaf et al., 2018 [17] & 0 & 1 & 3 & 2 & 1 & 7 & Good \\
\hline Messier et al., 2017 [18] & 2 & 1 & 1 & 2 & 1 & 7 & Good \\
\hline Nieman et al., 2013 [19] & 0 & 1 & 3 & 1 & 2 & 7 & Good \\
\hline Nieman et al., 2014 [20] & 0 & 1 & 3 & 1 & 2 & 7 & Good \\
\hline Ra et al., 2014 [21] & 2 & 1 & 1 & 2 & 1 & 7 & Good \\
\hline Stander et al., 2018 [22] & 2 & 1 & 1 & 2 & 1 & 7 & Good \\
\hline Howe et al., 2018 [24] & 0 & 0 & 3 & 2 & 1 & 6 & Good \\
\hline Neal et al., 2013 [25] & 0 & 1 & 1 & 2 & 2 & 6 & Good \\
\hline Peake et al., 2014 [26] & 0 & 2 & 1 & 1 & 2 & 6 & Good \\
\hline Pechlivanis et al., 2013 [27] & 0 & 1 & 1 & 2 & 2 & 6 & Good \\
\hline Zafeiridis et al., 2016 [28] & 0 & 1 & 1 & 2 & 2 & 6 & Good \\
\hline Muhsen Ali et al., 2016 [29] & 0 & 0 & 3 & 2 & 0 & 5 & Fair \\
\hline Castro et al., 2019 [30] & 2 & 1 & 1 & 1 & 0 & 5 & Fair \\
\hline Enea et al., 2010 [31] & 0 & 1 & 1 & 2 & 1 & 5 & Fair \\
\hline Andersson Hall et al., 2015 [32] & 0 & 1 & 1 & 2 & 1 & 5 & Fair \\
\hline Pechlivanis et al., 2010 [33] & 0 & 1 & 1 & 2 & 1 & 5 & Fair \\
\hline Wang et al., 2015 [34] & 0 & 1 & 1 & 2 & 1 & 5 & Fair \\
\hline Yan et al., 2009 [35] & 0 & 1 & 1 & 2 & 1 & 5 & Fair \\
\hline Prado et al., 2017 [36] & 2 & 0 & 2 & 0 & 0 & 4 & Fair \\
\hline Sun et al., 2017 [37] & 0 & 0 & 1 & 2 & 1 & 4 & Fair \\
\hline Berton et al., 2017 [38] & 0 & 1 & 1 & 0 & 1 & 3 & Poor \\
\hline Valério et al., 2018 [39] & 0 & 1 & 1 & 0 & 1 & 3 & Poor \\
\hline
\end{tabular}




\subsection{Exercise Intensity and Duration Effects on Metabolism}

Metabolic responses to exercise depend on the intensity and duration of effort. For the purposes of this review, heavy and moderate-intensity were differentiated using an intensity threshold of $60 \%$ of the oxygen uptake and heart rate reserve, and long and short-duration using a duration threshold of $60 \mathrm{~min}[40]$.

\subsection{High-Intensity and Long-Duration}

More than half of the studies included in this analysis $(62.5 \% ; n=15)$ measured metabolite responses to long-duration, high-intensity running $(n=8)[8,9,12-14,19,22,24]$, cycling $(n=5)[5,7,17,18,20]$, soccer $(n=1)$, and swimming $(n=1)$ [16] (Table 2). Liquid chromatography mass spectrometry (LC-MS) with or without GC-MS was used for metabolite identification in 11 [5,7-9,12-14,17,19,20,24] of these studies, with GC-MS as the primary method in two studies [16,22], capillary electrophoresis time-of-flight mass spectrometry (CE-TOFMS) in one study [21] and nuclear magnetic resonance (NMR) for one study [18]. Large-fold changes in metabolites from the lipid super pathway were reported by most investigators, including increases in plasma medium- and long-chain fatty acids, fatty acid oxidation products (dicarboxylate and monohydroxy fatty acids, acylcarnitines), and ketone bodies, with corresponding decreases in triacylglycerol esters (Table 2). Other metabolite changes included shifts in amino acids and increases in energy tricarboxylic acid (TCA) cycle components.

2.3. High-Intensity and Short-Duration, Moderate-Intensity and Short/Long-Duration, Cross-Sectional and Training Studies

(A) High-Intensity, Short-Duration

Two studies measured metabolite responses to high-intensity, short-duration (18 to $30 \mathrm{~min}$ ) exercise in recreationally active males and soccer athletes [23,28] (Table 3). Metabolite data from these studies were derived from GC-MS and NMR analytical platforms. Relatively small post-exercise changes were reported for metabolites related to the TCA cycle and related bioenergetics pathways. 
Table 2. High-intensity and long-duration studies.

\begin{tabular}{|c|c|c|c|c|c|}
\hline $\begin{array}{l}\text { Investigators Year } \\
\text { Published }\end{array}$ & Study Population & $\begin{array}{c}\text { Analytical } \\
\text { Platform/Matrix }\end{array}$ & Research Design & $\begin{array}{l}\text { Key Findings Exercise Effect Separate from Other } \\
\text { Interventions }\end{array}$ & Intensity \\
\hline Nieman et al., 2015 [5] & $\begin{array}{c}20 \text { male cyclists (aged } 39.2 \\
\pm 1.9 \text { years) }\end{array}$ & $\begin{array}{l}\text { UPLC-MS/MS; } \\
\text { Plasma }\end{array}$ & $\begin{array}{l}\text { Randomized, cross-over design; three trials of a } 75-\mathrm{km} \text { cycling } \\
\text { protocol ingesting: water only, bananas and water, pears and } \\
\text { water (2-week washout); blood samples timepoints: pre- and } \\
\text { post-exercise (0-h, 1.5-h, 21-h) }\end{array}$ & $\begin{array}{c}509 \text { metabolites were chemically identified; } \uparrow \text { ratio }>2 \text {-fold: } 107 \\
\text { metabolites increased in the water only trial (exercise effects); } \uparrow \\
\text { ratio }>5 \text {-fold: } 35 \text { metabolites increased, all from the lipid super } \\
\text { pathway, all significantly elevated 1.5-h post exercise, } 8 \text { only } \\
\text { remained after 21-h post-exercise. }\end{array}$ & $\begin{array}{l}\text { High-intensity, } \\
\text { long-duration }\end{array}$ \\
\hline Nieman et al., 2014* [7] & $\begin{array}{l}19 \text { male cyclists (aged } \\
38.06 \pm 1.6 \text { years) }\end{array}$ & $\begin{array}{l}\text { GC-MS and } \\
\text { UHPLC-MS/MS; } \\
\text { Plasma }\end{array}$ & $\begin{array}{l}\text { Randomized, cross-over design; two trials of a 75-km cycling } \\
\text { protocol with pistachio or no pistachio supplementation (2-week } \\
\text { washout); blood samples timepoints: pre- and post-exercise (0-h, } \\
1.5-\mathrm{h}, 21-\mathrm{h})\end{array}$ & $\begin{array}{l}423 \text { metabolites were chemically identified; Exercise increased } \\
167 \text { metabolites; All but } 2 \text { of these metabolites were related to } \uparrow \\
\text { lipid and carnitine metabolism, with the largest fold changes seen } \\
\text { for ketones, dicarboxylate fatty acids, and long chain fatty acids. }\end{array}$ & $\begin{array}{l}\text { High-intensity, } \\
\text { long-duration }\end{array}$ \\
\hline Nieman et al., 2017 [8] & $\begin{array}{c}24 \text { trained male runners } \\
\text { (aged } 36.5 \pm 1.8 \text { years) }\end{array}$ & $\begin{array}{l}\text { GC-MS and } \\
\text { UHPLC-MS/MS; } \\
\text { Plasma }\end{array}$ & $\begin{array}{l}\text { Repeated measures, ANOVA analysis, one group design; blood } \\
\text { samples collected pre- and post-exercise (0-h), one bout run to } \\
\text { exhaustion at } 70 \% \mathrm{VO}_{2 \max }\end{array}$ & $\begin{array}{l}209 \text { chemically identified metabolites changed with exercise, } \\
\text { especially long and medium-chain } \uparrow \text { fatty acids, } \uparrow \text { fatty acids } \\
\text { oxidation products (dicarboxylate and monohydroxy fatty acids } \\
\text { and acylcarnitines), and } \uparrow \text { ketone bodies. Minor relationship with } \\
\uparrow \text { IL- } 6 \text {. }\end{array}$ & $\begin{array}{l}\text { High-intensity, } \\
\text { long-duration }\end{array}$ \\
\hline Davison et al., 2018 [9] & $\begin{array}{l}24 \text { healthy males (aged } 28 \\
\pm 5 \text { years) }\end{array}$ & $\begin{array}{l}\text { LC-MS; } \\
\text { Serum }\end{array}$ & $\begin{array}{c}\text { Double-blind, randomized, cross-over design; 60-min run } 75 \% \\
\mathrm{VO}_{2 \text { max }} \text { in hypoxia }(\mathrm{FiO}=0.16 \% \text { ) (hypoxia chamber) and } \\
\left.\text { normoxia (FiO } \mathrm{F}_{2}=0.21 \%\right)(1-\text { week washout); blood samples } \\
\text { timepoints: pre- (after 30-min rest in hypoxia, normoxia), } \\
\text { post-exercise }(0 \mathrm{~h}, 3 \text {-h) }\end{array}$ & $\begin{array}{l}27 \text { metabolites, identified using internet databases, changed with } \\
\text { exercise; Most related to } \uparrow \text { lipid metabolism (several } \\
\text { acylcarnitines molecules identified) and purine metabolism } \\
\text { [†adenine, } \uparrow \text { adenosine and } \downarrow \text { ( } 3 \text { hafter recovery) hypoxanthine]; } \\
\uparrow 4.3 \text {-fold increase in } 18 \text { acylcarnitines post-exercise, above } \\
\text { pre-exercise at 3-h recovery. }\end{array}$ & $\begin{array}{l}\text { High-intensity, } \\
\text { long-duration }\end{array}$ \\
\hline Lehman et al., 2010 [12] & $\begin{array}{l}\text { Healthy subjects; } 1 \text { st } \\
\text { study: } n=13(32.6 \pm 6.1 \\
\text { years) 2nd study: } n=8 \\
\text { (30.9 } \pm 5.8 \text { years) }\end{array}$ & $\begin{array}{l}\text { UPLC-qTOF-MS; } \\
\text { Plasma }\end{array}$ & $\begin{array}{l}\text { Parallel group design; } 1 \text { st study: treadmill run } 60 \mathrm{~min} \text { at } 75 \% \mathrm{VO}_{2} \text {, } \\
\text { blood samples timepoints: pre- and post-exercise (0-h, 3-h, 24-h); } \\
\text { 2nd study: treadmill run > } 120 \mathrm{~min} \text { at } 70 \% \mathrm{~V}_{\text {IAT }} \text {, blood samples } \\
\text { timepoints: pre- (1h } 45 \text { min after breakfast) and post-exercise }(0-\mathrm{h}, \\
\text { 3-h, 24-h) }\end{array}$ & $\begin{array}{l}10 \text { metabolites, chemically identified, characterized the } \\
\text { separation between the timepoints; Most part non-esterified free } \\
\text { fatty acids; } \uparrow 9 \text {-fold increases in acylcarnitines. }\end{array}$ & $\begin{array}{l}\text { High-intensity, } \\
\text { long-duration }\end{array}$ \\
\hline Lewis et al., 2010 [13] & $\begin{array}{l}25 \text { amateur runners (aged } \\
42 \pm 9 \text { years) }\end{array}$ & $\begin{array}{l}\text { LC-MS; } \\
\text { Plasma }\end{array}$ & $\begin{array}{l}\text { Repeated measures, one group; Boston Marathon; blood samples } \\
\text { time points: pre- and post-marathon }\end{array}$ & $\begin{array}{l}\text { Metabolites chemically identified; } \uparrow \text { in glycolysis, lipolysis, } \\
\text { adenine nucleotide catabolism, and amino acid catabolism; } \uparrow \\
\text { indicators of glycogenolysis (glucose- } 6 \text {-phosphate and } \\
\text { 3-phosphoglycerate), and small molecules that reflect oxidative } \\
\text { stress (allantoin), and that modulate insulin sensitivity } \\
\text { (niacinamide) }\end{array}$ & $\begin{array}{l}\text { High-intensity, } \\
\text { long-duration }\end{array}$ \\
\hline Nieman et al., 2013 [14] & $\begin{array}{l}35 \text { long-distance male } \\
\text { runners (supplemented } \\
\text { group: aged } 33.7 \pm 6.8 \\
\text { years; placebo: aged } 35.2 \\
\quad \pm 8.7 \text { years) }\end{array}$ & $\begin{array}{l}\text { GC-MS and } \\
\text { UHPLC-MS/MS; } \\
\quad \text { Serum }\end{array}$ & $\begin{array}{c}\text { Double-blind, parallel group design; } 2 \text {-week supplementation } \\
\text { (polyphenol-enriched protein) followed by a 3-day intensified } \\
\text { exercise (2.5-h at } 70 \% \mathrm{VO}_{2 \text { max }} \text { bouts); blood samples timepoints: } \\
\text { pre- and post- 14-day supplementation, and immediately and } \\
\text { 14-h after the 3rd day of running }\end{array}$ & $\begin{array}{l}324 \text { chemically identified metabolites that changed with 3-day } \\
\text { period of exercise; } \uparrow \text { metabolites related to fatty acid oxidation } \\
\text { and ketogenesis including free fatty acids, acylcarnitines, } \\
\text { 3-hydroxy-fatty acids, and dicarboxylic acids, amino acid and } \\
\text { carbohydrate metabolism, energy production, nucleotides, and } \\
\text { cofactors and vitamins. }\end{array}$ & $\begin{array}{l}\text { High-intensity, } \\
\text { long-duration }\end{array}$ \\
\hline Knab et al., 2013 [16] & $\begin{array}{l}9 \text { elite male sprint and } \\
\text { middle-distance swim } \\
\text { athletes; } 7 \text { control subjects } \\
\text { (healthy and exercised } \\
\text { less than } 150 \mathrm{~min} / \text { week) } \\
\text { (aged } 24.6 \pm 0.7 \text { years) }\end{array}$ & $\begin{array}{l}\text { GC-MS; } \\
\text { Serum }\end{array}$ & $\begin{array}{l}\text { Randomized, crossover design, 10-day supplementation with } \\
\text { juice ( } 8 \text { fl oz pre- and post-training) or non-juice, 10-d practice of } \\
\text { 2-h swimming, approximately 5500-m swim interval training } \\
\text { (3-week washout). Blood samples timepoints: pre- and post- } \\
\text { each 10-days supplementation period and post-exercise (0-h) }\end{array}$ & $\begin{array}{l}325 \text { metabolites were chemically identified; No effects of juice on } \\
\text { exercise-induced measures; } \uparrow \text { Oxidative stress and } \downarrow \text { antioxidant } \\
\text { capacity in swimmers group compared to nonathletic control } \\
\text { grou; M Metabolites that differed mostly related to substrate } \\
\text { utilization and supplements used by the swimmers. Pre and } \\
\text { post-exercise small but significant shift in metabolites related to } \\
\text { substrate utilization: pyruvic acid, propanoic acid, d-fructose, } \\
\text { mannose, n-acetylglutamine, norleucine, alloisoleucine, and } \\
\text { d-glucuronic acid. }\end{array}$ & $\begin{array}{l}\text { High-intensity, } \\
\text { long-duration }\end{array}$ \\
\hline
\end{tabular}


Table 2. Cont.

\begin{tabular}{|c|c|c|c|c|c|}
\hline $\begin{array}{l}\text { Investigators Year } \\
\text { Published }\end{array}$ & Study Population & $\begin{array}{c}\text { Analytical } \\
\text { Platform/Matrix }\end{array}$ & Research Design & $\begin{array}{l}\text { Key Findings Exercise Effect Separate from Other } \\
\text { Interventions }\end{array}$ & Intensity \\
\hline Manaf et al., 2018 [17] & $\begin{array}{l}18 \text { healthy and } \\
\text { recreationally active males } \\
\text { (aged } 24.7 \pm 4.8 \text { years) }\end{array}$ & $\begin{array}{l}\text { LC-MS; } \\
\text { Plasma }\end{array}$ & $\begin{array}{l}\text { Repeated measures, ANOVA analysis, one group design; } \\
\text { time-to-exhaustion (81-min) cycling test at a workload } 3 \mathrm{mM} / 1 \\
\text { lactate; blood samples timepoints: pre-exercise, during exercise } \\
\text { (10-min, before fatigue), point of exhaustion (immediately after } \\
\text { fatigue), post-exercise (20-min after fatigue) }\end{array}$ & $\begin{array}{l}80 \text { metabolites identified using internet databases; } 68 \text { metabolites } \\
\text { changed during exercise; } \uparrow \text { Free-fatty acids and } \downarrow \text { tryptophan } \\
\text { contributed to differences in plasma metabolome at fatigue. }\end{array}$ & $\begin{array}{l}\text { High-intensity, } \\
\text { long-duration }\end{array}$ \\
\hline Messier et al., 2017 [18] & $\begin{array}{c}20 \text { healthy male (aged } 39 \\
\pm 4.3 \text { years) }\end{array}$ & $\begin{array}{l}\text { 1H NMR; } \\
\text { Plasma }\end{array}$ & $\begin{array}{l}\text { Cross-over design; cycling 60-min at ventilatory threshold } 1 \text { at } 70 \\
\text { rpm, at sea level and above } 2150 \mathrm{~m} \text { of the sea level ( } 2 \text {-week } \\
\text { washout); blood samples timepoints: pre- and post-exercise (0-h) }\end{array}$ & $\begin{array}{l}18 \text { metabolites identified using internet databases; } \downarrow \text { glucose and } \\
\text { free amino acid levels; No differences in lipid metabolism } \\
\text { between altitudes; Fuel shift from lipid oxidation to carbohydrate } \\
\text { oxidation at } 2150 \text { above sea level. }\end{array}$ & $\begin{array}{l}\text { High-intensity, } \\
\text { long-duration }\end{array}$ \\
\hline Nieman et al., 2013 [19] & $\begin{array}{c}15 \text { runners }(7 \text { males, } 8 \\
\text { females) (aged } 35.2 \pm 8.7 \\
\text { years) }\end{array}$ & $\begin{array}{l}\text { GC-MS and } \\
\text { UHPLC-MS/MS; } \\
\text { Serum }\end{array}$ & $\begin{array}{l}\text { Cross-sectional design, 3-day period exercise }(2.5 \mathrm{~h} \text { per day } \\
\left.\text { running bouts at approximately } 70 \% \mathrm{VO}_{2 m a x}\right) ; \text { blood samples } \\
\text { timepoints: pre- and post-exercise }(0-\mathrm{h}, 14-\mathrm{h})\end{array}$ & $\begin{array}{l}\text { Metabolites chemically identified; } \uparrow \geq 2 \text {-fold increases in } 75 \\
\text { metabolites immediately post } 3 \text {-day exercise period, } 22 \text { related to } \\
\text { lipid/carnitine metabolism, } 13 \text { related to amino acid/peptide } \\
\text { metabolism, } 4 \text { to hemoglobin/porphyrin metabolism, and } 3 \text { to } \\
\text { Krebs cycle intermediates. After } 14 \text {-h recovery: } 50 \text { of } 75 \\
\text { metabolites still elevated. } \downarrow 22 \text { metabolites post-exercise related } \\
\text { to lysolipid and bile acid metabolism. }\end{array}$ & $\begin{array}{l}\text { High-intensity, } \\
\text { long-duration }\end{array}$ \\
\hline Nieman et al., 2014* [20] & $\begin{array}{l}19 \text { male cyclists (aged } \\
38.06 \pm 1.6 \text { years) }\end{array}$ & $\begin{array}{l}\text { GC-MS and } \\
\text { UHPLC-MS/MS; } \\
\text { Plasma }\end{array}$ & $\begin{array}{l}\text { Repeated measures, ANOVA analysis, one group design; blood } \\
\text { samples timepoints: pre- and post-exercise (0-h, 1.5-h, 21-h); } \\
\text { 75-km cycling protocol }\end{array}$ & $\begin{array}{l}221 \text { chemically identified metabolites changed with exercise; all } \\
\text { but } 26 \text { related to } \uparrow \text { lipid and carnitine metabolism; largest fold } \\
\text { changes seen for } \uparrow \text { ketones, dicarboxylate fatty acids, and long } \\
\text { chain fatty acids. }\end{array}$ & $\begin{array}{l}\text { High-intensity, } \\
\text { long-duration }\end{array}$ \\
\hline Ra et al., 2014 [21] & $\begin{array}{l}37 \text { male soccer players } \\
\text { (aged } 20.6 \pm 0.04 \text { years) }\end{array}$ & $\begin{array}{l}\text { CE-TOFMS; } \\
\text { Saliva }\end{array}$ & $\begin{array}{l}\text { Repeated measures, ANOVA analysis, one group design; 3-day } \\
\text { game program (90-min per day); saliva samples timepoints: } \\
\text { pre-exercise (1-month before) and post-exercise (24-h after) }\end{array}$ & $\begin{array}{l}144 \text { metabolites chemically identified; } \uparrow 12 \text { metabolites (e.g., } \\
\text { 3-methylhistidine, glucose } 1 \text { - and } 6 \text {-phosphate, taurine, amino } \\
\text { acids) related to muscle catabolism, glucose metabolism, lipid } \\
\text { metabolism, amino acid metabolism and energy metabolism. }\end{array}$ & $\begin{array}{l}\text { High-intensity, } \\
\text { long-duration }\end{array}$ \\
\hline Stander et al., 2018 [22] & $\begin{array}{l}31 \text { recreational marathon } \\
\text { athletes (19 males and } 12 \\
\text { females) (aged } 41 \pm 12 \\
\text { years) }\end{array}$ & $\begin{array}{l}\text { GC-TOFMS; } \\
\text { Serum }\end{array}$ & $\begin{array}{l}\text { Repeated measures, ANOVA analysis, one group design; } 42-\mathrm{km} \\
\text { marathon; blood samples timepoints: pre- and post-marathon } \\
(0-\mathrm{h})\end{array}$ & $\begin{array}{l}70 \text { metabolites chemically identified; } \uparrow \text { carbohydrates, fatty acids, } \\
\text { tricarboxylic acid cycle intermediates, ketones, and } \downarrow \text { amino acids; } \\
\text { } \text { add-chain fatty acids and } \alpha \text {-hydroxy acids. }\end{array}$ & $\begin{array}{l}\text { High-intensity, } \\
\text { long-duration }\end{array}$ \\
\hline Howe et al., 2018 [24] & $\begin{array}{l}9 \text { male ultramarathon } \\
\text { runners (aged } 34 \pm 7 \\
\text { years) }\end{array}$ & $\begin{array}{l}\text { HILIC-MS; } \\
\text { Plasma }\end{array}$ & $\begin{array}{l}\text { Repeated measures, ANOVA analysis, one group design; 80.5-km } \\
\text { treadmill simulated ultramarathon run; blood samples } \\
\text { timepoints: pre- and post-exercise (0-h) }\end{array}$ & $\begin{array}{l}446 \text { metabolites chemically identified; } \downarrow \text { amino acids metabolism } \\
\text { post- } 80.5 \mathrm{~km} ; \uparrow \text { in the formation of medium-chain unsaturated, } \\
\text { partially oxidized fatty acids and conjugates of fatty acids with } \\
\text { carnitines. }\end{array}$ & $\begin{array}{l}\text { High-intensity, } \\
\text { long-duration }\end{array}$ \\
\hline
\end{tabular}

UPLC-MS: ultra-performance liquid chromatography mass spectrometry; UHPLC-MS: ultra-high-performance liquid chromatography mass spectrometry; GC-MS: gas chromatography mass spectrometry; LC-MS: liquid chromatography mass spectrometry; UHPLC/Q-TOF MS: ultra-high-performance liquid chromatography quadrupole time-of-flight mass spectrometry; 1H NMR: proton nuclear magnetic resonance; CE-TOFMS: capillary electrophoresis time-of-flight mass spectrometry; HILIC-MS: hydrophilic interaction chromatography mass spectrometry; $\mathrm{VO}_{2 \max }=$ maximal oxygen uptake; $\mathrm{FiO}_{2}=$ fraction of inspired oxygen; $\mathrm{V}_{\mathrm{IAT}}=$ velocity at individual anaerobic threshold. ${ }^{*}$ References $[7,20]$ were from the same study but the data sets provided additive information. 
Table 3. Summaries of study characteristics and findings from nine $[6,10,11,15,23,25-28]$ studies using other types of exercise designs.

\begin{tabular}{|c|c|c|c|c|c|}
\hline $\begin{array}{l}\text { Investigators Year } \\
\text { Published }\end{array}$ & Study Population & $\begin{array}{l}\text { Analytical } \\
\text { Platform/Matrix }\end{array}$ & Research Design & $\begin{array}{l}\text { Key Findings Exercise Effect Separate from Other } \\
\text { Interventions }\end{array}$ & Intensity \\
\hline Danaher et al., 2015 [23] & $\begin{array}{l}7 \text { active males (aged } 22.9 \\
\pm 5.0 \text { years) }\end{array}$ & $\begin{array}{l}\text { GC-MS; } \\
\text { Plasma }\end{array}$ & 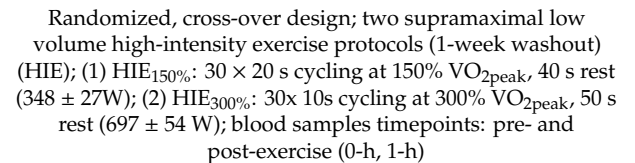 & $\begin{array}{l}55 \text { chemically identified metabolites detected; HIE300\% } \\
\text { produced greater metabolic perturbations compared to } \\
\text { HIE150\%; Changes more pronounced during recovery than } \\
\text { exercise, with } \uparrow \text { glycolytic pathway and fatty acids and lipid } \\
\text { metabolism. }\end{array}$ & $\begin{array}{l}\text { High-intensity, } \\
\text { short-duration }\end{array}$ \\
\hline Zafeiridis et al., 2016 [28] & $\begin{array}{c}9 \text { healthy young men } \\
\text { (aged } 20.5 \pm 0.7 \text { years). } \\
\text { Soccer training } 4-5 \text { times } \\
\text { per week. }\end{array}$ & $\begin{array}{l}\text { 1H NMR; } \\
\text { Plasma }\end{array}$ & $\begin{array}{l}\text { Randomized, cross-over design; three running protocols } \\
\text { (2-week washout): intense continuous (18-min, } 80 \% \text { of } \\
\text { maximum aerobic velocity (MAV)), long-interval (29-min, } 3 \\
\text { min at } 95 \% \text { of MAV, } 3 \text { min recovery at } 35 \% \text { of MAV and } \\
\text { short-interval (18-min, } 30 \mathrm{~s} \text { at } 110 \% \text { of MAV, } 30 \mathrm{~s} \text { recovery at } \\
50 \% \text { of MAV); blood sample timepoints: pre- and post-exercise } \\
\text { (5-min). }\end{array}$ & $\begin{array}{l}17 \text { metabolites identified using internet databases; } \\
\text { No detectable difference in metabolites; } \uparrow \text { carbohydrate/lipid } \\
\text { metabolism and activation of the TCA cycle in all three } \\
\text { protocols. }\end{array}$ & $\begin{array}{l}\text { High-intensity, } \\
\text { short-duration }\end{array}$ \\
\hline Jacobs et al., 2014 [6] & $\begin{array}{c}19 \text { healthy physically } \\
\text { active males (aged } 21 \pm 2 \\
\text { years) }\end{array}$ & $\begin{array}{l}\text { GC-MS and LC-MS/MS; } \\
\text { Plasma }\end{array}$ & $\begin{array}{l}\text { Double-blind, randomized, cross-over design; } 6 \text {-day } \\
\text { supplementation with decaffeinated green tea or placebo } \\
\text { ingestion (28-day washout) 2-h before a } 30 \text { min cycle exercise at } \\
55 \% \text { Vo2max }\end{array}$ & $\begin{array}{l}152 \text { chemically identified metabolites changed with exercise; } \uparrow \\
\text { metabolites related to adrenergic and energy metabolism (e.g., } \\
\text { lactate, pyruvate, malate, succinate, glycerol, cortisol); } \downarrow \\
\text { 2-hydrxobutyrate. }\end{array}$ & $\begin{array}{l}\text { Moderate-intensity, } \\
\text { short-duration }\end{array}$ \\
\hline Hodgson et al., 2012 [10] & $\begin{array}{l}27 \text { healthy physically } \\
\text { active males (aged } 22 \pm 5 \\
\text { years) }\end{array}$ & $\begin{array}{l}\text { GC-MS and LC-MS/MS; } \\
\text { Plasma }\end{array}$ & $\begin{array}{l}\text { Double-blind, randomized, parallel design; } 7 \text {-day } \\
\text { supplementation with caffeinated green tea or placebo } \\
\text { ingestion 2-h before 60-min cycle exercise at } 50 \% \mathrm{VO}_{2 \max }\end{array}$ & $\begin{array}{l}238 \text { metabolites chemically detected changed with exercise; } \uparrow \\
\text { ratio > 2: lactate, pyruvate, succinate, noradrenaline and } \\
\text { glyceroli } \downarrow 2 \text { 2-hydroxybutyrate, trans-4-hydroxyproline, } \\
\text { mannose, certain triacylglycerides (TAGs) and nicotinamide. } \\
478 \text { chemically identified metabolites changed pre- and }\end{array}$ & $\begin{array}{l}\text { Moderate-intensity, } \\
\text { long-duration }\end{array}$ \\
\hline Karl et al., 2017 [11] & $\begin{array}{l}25 \text { male highly trained } \\
\text { soldiers (aged } 19.0 \pm 1.0 \\
\text { years) }\end{array}$ & $\begin{array}{l}\text { UPLC-MS/MS; } \\
\text { Plasma }\end{array}$ & $\begin{array}{l}\text { Double-blind, randomized, parallel design; } 4 \text {-day, } 51-\mathrm{km} \\
\text { cross-country ski march carrying } 45 \mathrm{~kg} \text { pack; blood sample } \\
\text { timepoints: pre- and post-exercise (early completers: } 8 \text { to } 10-\mathrm{h} \\
\text { or late completers: } 2 \text { to } 3 \text {-h). }\end{array}$ & $\begin{array}{l}\text { post-exercise } \uparrow 88 \% \text { of the free fatty acids; } \uparrow 91 \% \text { of the } \\
\text { acylcarnitines; } \downarrow 88 \% \text { of the mono- and diacylglycerols detected } \\
\text { within lipid metabolism pathways; Smaller } \uparrow 75 \% \text { of the } \\
\text { tricarboxylic acid cycle intermediates; } \uparrow 50 \% \text { of the branched } \\
\text { chain amino acid metabolites }\end{array}$ & $\begin{array}{l}\text { Moderate-intensity, } \\
\text { long-duration }\end{array}$ \\
\hline Peake et al., 2014 [26] & $\begin{array}{l}10 \text { well-trained male } \\
\text { cyclists and triathletes } \\
\text { (aged } 33.2 \pm 6.7 \text { years) }\end{array}$ & $\begin{array}{l}\text { GC-MS; } \\
\text { Plasma }\end{array}$ & $\begin{array}{l}\text { Randomized, cross-over design; } \mathrm{HITT}\left(60-\mathrm{min}, \approx 82 \% \mathrm{VO}_{2 \max },\right) \\
\text { and a moderate-intensity continuous exercise }(\mathrm{MOD})(61-\mathrm{min}, \\
\left.\approx 67 \% \mathrm{VO}_{2 \text { max }}\right) \text {; blood samples timepoints: pre- and } \\
\text { post-exercise }(0-\mathrm{h}, 1-\mathrm{h}, 2 \text {-h). }\end{array}$ & $\begin{array}{l}49 \text { metabolites chemically identified; } 29 \text { changed with exercise } \\
\text { (11 changed with both HIIT and MOD; } 13 \text { changed with HIIT } \\
\text { only; } 5 \text { changed with MOD only); } \uparrow \text { in carbohydrate oxidation } \\
\text { and } \downarrow \text { in fat oxidation in HIIT exercise compared to MOD; } \\
\text { Glucose and lactate higher at } 0 \text {-h in HIIT compared to MOD. }\end{array}$ & $\begin{array}{l}\text { High and } \\
\text { moderate-intensity, } \\
\text { long-duration }\end{array}$ \\
\hline Al-Khelaifi et al., 2018 [15] & $\begin{array}{l}191 \text { elite athletes }(171 \\
\text { males, } 20 \text { females })\end{array}$ & $\begin{array}{l}\text { UPLC-MS/MS; } \\
\text { Serum }\end{array}$ & $\begin{array}{l}\text { Cross-sectional design using elite athletes from various sport } \\
\text { disciplines being monitored for doping; blood samples } \\
\text { collected IN or OUT competition (1 timepoint) }\end{array}$ & $\begin{array}{c}\text { Metabolites chemically identified; } \uparrow \text { Oxidative stress common } \\
\text { to both high-power and high-endurance sports alike; } \uparrow \text { steroids } \\
\text { and polyamine pathways more prominent in endurance; } \uparrow \\
\text { sterols, adenine-containing purines, and energy metabolites } \\
\text { most evident with power. }\end{array}$ & Cross-section elite athletes \\
\hline Neal et al., 2013 [25] & $\begin{array}{l}12 \text { male cyclists (aged } 36 \pm \\
6 \text { years) }\end{array}$ & $\begin{array}{l}\text { 1H NMR; } \\
\text { Urine }\end{array}$ & $\begin{array}{c}\text { Randomized, cross-over design; 6-week training of polarized } \\
\text { training-intensity }(80 \% \text { low intensity, } 0 \% \text { moderate-intensity, } \\
20 \% \text { high-intensity) and a training-intensity distribution (57\% } \\
\text { low intensity, } 43 \% \text { moderate-intensity, 0\% high-intensity) } \\
\text { (4-week washout); urine samples timepoints: pre- and post- } \\
\text { each training period. }\end{array}$ & $\begin{array}{l}\text { Method used to identify metabolites not reported; metabolites } \\
\text { identified as } \downarrow \text { hippuric acid, } \uparrow \text { creatinine, } \uparrow \text { dimethylamine, } \uparrow \\
\text { 3-methylxanthine, } \downarrow \text { hypoxanthine. }\end{array}$ & $\begin{array}{l}\text { Chronic training, Low, } \\
\text { moderate and } \\
\text { high-intensity }\end{array}$ \\
\hline
\end{tabular}


Table 3. Cont

\begin{tabular}{ccccc}
\hline $\begin{array}{c}\text { Investigators Year } \\
\text { Published }\end{array}$ & Study Population & $\begin{array}{c}\text { Analytical } \\
\text { Platform/Matrix }\end{array}$ & Research Design & Key Findings Exercise Effect Separate from Other \\
Interventions
\end{tabular}

GC-MS: gas chromatography mass spectrometry; LC-MS: liquid chromatography mass spectrometry; UPLC-MS: ultra-performance liquid chromatography mass spectrometry; 1H NMR: proton nuclear magnetic ressonance; UPLC-MS: ultra-performance liquid chromatography mass spectrometry; HIE = high-intensity exercise; HIE150\% $=$ high-intensity exercise at $150 \%$ of VO2 peak; $\mathrm{HIE} 300 \%$ = high-intensity exercise at $300 \%$ of VO2 peak; $\mathrm{W}$ = watts; MAV = maximum aerobic velocity; TCA = tricarboxylic acid cycle; HIIT: high-intensity interval training; MOD = moderate-intensity continous exercise. 
(B) Moderate-Intensity, Short-Duration

One study reported metabolite responses following moderate-intensity, short-duration (30 min) cycling [6] (Table 3). Metabolites were identified using GC-MS and LC-MS/MS analytical platforms. Small-fold post-exercise changes were reported for metabolites linked to energy metabolism (lipolysis, glycolysis, TCA cycle intermediates, and catecholamines).

(C) Moderate-Intensity, Long-Duration

Two studies investigated metabolite responses to moderate-intensity, long-duration cycling and cross-country skiing $[10,11]$. Small to moderate post-exercise changes were reported for metabolites related to glycolytic and lipid pathways including free fatty acids, branched chain amino acids, acylcarnitines, mono- and diacylglycerols, and TCA intermediates.

(D) High- and Moderate-Intensity, Long-Duration

One study compared metabolite responses of high-intensity interval training (HIIT) and $60 \mathrm{~min}$ of moderate-intensity cycling (MOD) using GC-MS [26]. Small- to moderate-fold changes were reported for metabolites related to energy metabolism, and glycolytic and lipid pathways. HIIT compared to MOD induced higher post-exercise levels for glycolytic-related metabolites, with lower levels of lipid related metabolites.

\section{(E) Cross-Section Elite Athletes}

One study compared plasma metabolite levels in athletes from high- $(n=121)$ and moderate$(n=70)$ endurance sports [15] (Table 3). Metabolomics was performed by ultra-performance liquid chromatography mass spectrometry (UPLC-MS/MS). The cross-sectional analysis showed some group differences, including higher levels for metabolites related to oxidative stress, fatty acid metabolism, steroid biosynthesis, and energy metabolism in high power and high endurance athletes. Plasma levels of metabolites related to steroid and polyamine pathways were more prominent in endurance athletes, with sterols, adenine-containing purines, and energy metabolites more evident in power athletes.

(F) Chronic Training, Low-, Moderate-, and High-Intensity

One study compared the chronic effects of cycling training at different intensities [25]. Plasma metabolites were measured with NMR, and only small group differences were reported in a few selected metabolites (hippuric acid, hypoxanthine, creatinine, dimethylamine, 3-methylxanthine).

(G) Chronic Training, High-Intensity

One study investigated the effects of chronic, high-intensity, short-duration running on plasma metabolite levels using NMR [27]. Small changes in selected metabolites were reported including lactate, pyruvate, TCA intermediates, and phospholipids.

\section{Discussion}

Advances in mass spectrometry since 2010 have led to an increasing number of metabolomics-based studies targeted on whole-body metabolite responses to varying acute and chronic exercise workloads. This systematic review of 24 high-quality papers published during the past decade revealed that the primary focus (63\% of studies) has been on acute metabolite perturbations to long-duration, high-intensity aerobic exercise. Little information is available regarding metabolite changes coupled to acute bouts of exercise with lower workload volumes or those linked to long-term exercise training. The best studies utilized LC-MS/MS analytical platforms with large chemical standards libraries to identify and detect exercise-induced shifts in hundreds of metabolites. Strong bioinformatics support has improved predictive and descriptive modelling, discriminative variable selection, and the overall understanding of the body's metabolome response to exercise. 
This review indicates that a bout of prolonged and intensive exercise causes large-fold changes in numerous and diverse lipid-related metabolites [5,7-9,12,14,17,19-22,24]. In a typical study with human athletes exercising intensely for more than two hours, significant increases in at least 300 identified metabolites can be measured by LC-MS/MS analytical platforms, with more than 100 increasing twofold or greater $[5,7,14,20,24]$. This response includes post-exercise increases in plasma medium- and long-chain fatty acids, ketone bodies, fatty acid oxidation products, and sulfated bile acids. At the same time, related decreases occur in plasma triacylglycerol esters, primary and secondary bile acids, and minor phospholipids such as lysophosphatidylcholines and lysophosphatidylethanolamines [12,19,20,41]. Untargeted metabolomics has revealed post-exercise increases in both common (e.g., oleate/vaccinate, palmitate, linoleate, stearate, palmitoleate, myristate), and atypical fatty acids (adrenate, docosapentaenoate, dihomo-linolenate, dihomolinoleate, docosadienoate, and eicosenoate). The corresponding fatty acid oxidation signature includes acylcarnitines, 3-hydroxybutyrate (BHBA), and dicarboxylate and monohydroxy fatty acids. Other important shifts have been measured for plasma concentrations of tryptophan- and other amino acid-related metabolites, and energy tricarboxylic acid (TCA) cycle components including malate, aconitate, citrate, fumarate, succinate, and alpha-ketoglutarate [13,16-19,22,41].

Most of the changes in plasma metabolites after prolonged and intensive exercise reach their nadir within a few hours. Plasma deviations in many of these metabolites are still apparent, but largely abated, after one day of recovery [5,7,12,19-21]. The large and varied metabolite response to heavy exercise workloads reflects the physiological stress and diminished glycogen stores experienced by the participant $[12,21,24,41]$.

An increasing number of studies are utilizing metabolomics to measure the influence of various nutritional interventions on metabolite perturbations during recovery from prolonged and intensive exercise $[5,7,14,16,41,42]$. Metabolomics is ideally suited to measuring the impact of nutritional interventions during acute exercise by simultaneously measuring and identifying shifts in hundreds of metabolites from diverse pathways. Emerging data indicate that carbohydrates from both sugar beverages and fruits such as bananas, and flavonoids from food and beverage sources such as blueberries and green tea, have a large effects on the human metabolome response to intense exercise workloads [3,5,14,16,42,43].

Relatively few studies have investigated exercise-induced metabolite changes following acute bouts with lower durations ( $<60 \mathrm{~min}$ ) and workload volumes [23,26]. Half of these studies performed metabolomics using GC-MS or NMR analytical platforms, limiting the number of identified metabolites and the usefulness of these data. As expected, post-exercise shifts in plasma metabolite levels are modest in comparison to high exercise volume workloads due to a moderated reliance on underlying carbohydrate and lipid substrate pathways.

More cross-sectional studies are needed to compare plasma and urine metabolite levels between sedentary and physically active individuals, and athletes from different sports. These studies could provide important information for future randomized, exercise training trials. Using a cross-sectional design, one study showed some metabolite differences between power and endurance athletic groups [15]. The athletes were not tested at the same time or in similar resting states, however, making it difficult to draw definitive conclusions.

Few randomized, exercise training studies have been conducted to investigate potential adaptations in the human metabolome $[25,27]$. These two studies employed different training protocols and study designs, and performed metabolomics using NMR, limiting the usefulness of these data. Future metabolomics-based randomized exercise training studies, especially when combined with genomics and proteomics outcomes, will improve scientific understanding of the human system's response to varying exercise workloads [44]. 


\section{Materials and Methods}

This systematic review was performed according to the Preferred Reporting Items for Systematic Reviews and Meta-Analyses (PRISMA) guidelines [45] and was preregistered in the International Prospective Register of Systematic Review (PROSPERO). To systematize the search and data extraction, a free standardized electronic tool called State of the Art through Systematic Review (StArt) [46] was used. The software StArt tracked duplicated studies during extraction, and this was confirmed with manual examination by the two main reviewers. The studies were selected, extracted and included independently by two researchers (CAS and EFS), and a third independent researcher (RMA) verified the inclusion process in order to solve any disagreement between the two main researchers.

\subsection{Search Strategy}

The electronic search was performed from inception to November 26th, 2018 and updated on April 10th, 2019. The articles were retrieved from the following electronic databases: PubMed (via National Library of Medicine), Science Direct, SCOPUS (Elsevier) and Web of Science. The MeSH terms were selected and combined according to analysis method (metabolomics) and mandatory activity (sports OR exercise). Moreover, the search strategy was limited to humans (population of interest), English language and clinical trial studies.

\subsection{Eligibility Criteria for Inclusion}

The abstracts were first examined and evaluated for the listed criteria. Studies were selected if metabolomics were utilized to measure exercise-induced changes in metabolites in healthy study participants using serum, plasma, saliva, or urine samples. Exercise-based studies with nutrition interventions were included, but this review only included data collected from the control groups. Reviews, case reports, guidelines, theses and dissertations, conference abstracts, and studies using animal or in vitro models were not included.

\subsection{Data Extraction and Study Inclusion}

The following data from the selected studies were extracted: name of the first author, year of publication, characteristics of participants and groups (population, sample size, groups, gender, age, physical activity level), research design elements (type of research, exercise mode, duration, and intensity), metabolomics procedures (analytical platform, metabolite data), and summary comments.

\subsection{Studies Quality Assessment}

The quality of the studies was assessed by two researchers (CAS and EFS) using a scoring system created for this analysis (see Table 4 and Figure 2).

\begin{tabular}{|c|c|}
\hline Total Score & Classification \\
\hline 9 to 11 & Excellent \\
6 to 8 & Good \\
4 to 5 & Fair \\
Below 4 & Poor \\
\hline
\end{tabular}

Figure 2. Classification of studies based on the total score. 
Table 4. Score setting for metabolomic studies quality assessment.

\begin{tabular}{|c|c|c|c|}
\hline \multicolumn{4}{|c|}{ Score Setting } \\
\hline Section & Maximum Score & Aspects & Score Attribution \\
\hline \multirow[t]{2}{*}{ Research Design } & 2 & Number of Participants & $\begin{array}{c}\text { Parallel Studies } \\
0-\mathrm{N}<20 \\
2-\mathrm{N}>20 \\
\text { Crossover Studies } \\
0-\mathrm{N}<13 \\
2-\mathrm{N}>13\end{array}$ \\
\hline & 2 & Study Characteristics & $\begin{array}{c}\text { Randomized control group } \\
\text { Proper matrix } \\
>2 \text { timepoints data collection } \\
\text { Duration } \geq 3 \text { week (chronic studies only) } \\
\text { 0-None of the previous items } \\
\text { 1-At least } 2 \text { of the first } 3 \text { criteria listed } \\
\text { 3-All } 3 \text { of the first } 3 \text { criteria listed }\end{array}$ \\
\hline \multirow[t]{2}{*}{ Methodology } & 3 & Analysis Methods & $\begin{array}{c}\text { 3-LC-MS/MS with extensive standards } \\
\text { 1-NMR 1H, limited standards } \\
\text { 1-GC-MS, limited standards }\end{array}$ \\
\hline & 2 & Statistical Support & $\begin{array}{c}\text { 0-simple univariate statistics } \\
\text { 1-Univariate statistics + additional } \\
\text { analyses to sort and group the data, and to } \\
\text { control for confounding factors } \\
\text { 2-Univariate statistics + PCA, OPLS-DA, } \\
\text { PLS-DA, or similar advanced } \\
\text { bioinformatics procedures }\end{array}$ \\
\hline Novelty & 2 & & New information in the literature \\
\hline
\end{tabular}

\section{Conclusions and Future Directions}

The first decade of metabolomics-based exercise studies, especially those utilizing sensitive LC-MS/MS analytical platforms with large chemical standards libraries and rigorous bioinformatics support, provided useful systems biology information on the biochemical mechanisms underlying exercise-induced effects on metabolism $[13,47]$. This area of scientific endeavor is still emerging, and much remains to be discovered, especially in the areas of the metabolite response to acute and chronic moderate exercise workloads. The sensitivity of the analytical platforms will continue to improve, expanding the number of small molecule metabolites that can be detected. These improvements in technology, coupled with improved quality control, bioinformatics support, the expansion of biochemical standards, and an emphasis on larger study groups of both genders, will improve the identification and quantitation of currently known and unknown metabolites in a variety of human matrixes. More emphasis is needed on the influence of activity reduction and physical inactivity on metabolite shifts. These improvements in study design and methodology will broaden our understanding of the influence of acute and chronic exercise on the human metabolome. An increasing number of studies, including the National Institutes of Health project, 'Molecular Transducers of Physical Activity in Humans', will combine metabolomics with genetics, epigenetics, lipidomics, and proteomics to examine all aspects of the physiological, biochemical, and molecular response to both aerobic- and resistance-based exercise training interventions [44].

Author Contributions: C.A.S., D.C.N. and A.M.C. organized the study and created the scoring and classification system; C.A.S., D.C.N., E.F.S., R.M.A. searched, extracted the studies from the databases and selected for inclusion; D.C.N., C.A.S., E.F.S. wrote; D.C.N., C.A.S., E.F.S., R.M.A., A.M.C. edited and reviewed the study.

Funding: São Paulo Research Fundation-FAPESP (grant \#2016/222157).

Acknowledgments: The authors would like to acknowledge the Coordenação de Aperfeiçoamento de Pessoal de Nível Superior, Brazil (CAPES, Postgraduate Program in Physiotherapy, grant: 001).

Conflicts of Interest: Authors declare no conflict of interest. 


\section{References}

1. Grazioli, E.; Dimauro, I.; Mercatelli, N.; Wang, G.; Pitsiladis, Y.; Di Luigi, L.; Caporossi, D. Physical activity in the prevention of human diseases: Role of epigenetic modifications. BMC Genom. 2017, 18, 802. [CrossRef] [PubMed]

2. Wackerhage, H.; Smith, J.; Wisniewski, D. Molecular Exercise Physiology, 1st ed.; Oxford University Press: Oxford, UK, 2014; p. 1. ISBN 978-0-19-181751-9.

3. Nieman, D.C.; Mitmesser, S.H. Potential impact of nutrition on immune system recovery from heavy exertion: A metabolomics perspective. Nutrients 2017, 9, 513. [CrossRef] [PubMed]

4. Dunn, W.B.; Broadhurst, D.I.; Atherton, H.J.; Goodacre, R.; Griffin, J.L. Systems level studies of mammalian metabolomes: The roles of mass spectrometry and nuclear magnetic resonance spectroscopy. Chem. Soc. Rev. 2011, 40, 387-426. [CrossRef] [PubMed]

5. Nieman, D.C.; Gillitt, N.D.; Sha, W.; Meaney, M.P.; John, C.; Pappan, K.L.; Kinchen, J.M. Metabolomics-based analysis of banana and pear ingestion on exercise performance and recovery. J. Proteome Res. 2015, 14, 5367-5377. [CrossRef] [PubMed]

6. Jacobs, D.M.; Hodgson, A.B.; Randell, R.K.; Mahabir-Jagessar-T, K.; Garczarek, U.; Jeukendrup, A.E.; Mela, D.J.; Lotito, S. Metabolic response to decaffeinated green tea extract during rest and moderate-intensity exercise. J. Agric. Food Chem. 2014, 62, 9936-9943. [CrossRef] [PubMed]

7. Nieman, D.C.; Scherr, J.; Luo, B.; Meaney, M.P.; Dréau, D.; Sha, W.; Dew, D.A.; Henson, D.A.; Pappan, K.L. Influence of pistachios on performance and exercise-induced inflammation, oxidative stress, immune dysfunction, and metabolite shifts in cyclists: A randomized, crossover trial. PLoS ONE 2014, 9, e113725. [CrossRef] [PubMed]

8. Nieman, D.C.; Sha, W.; Pappan, K.L. IL-6 linkage to exercise-induced shifts in lipid-related metabolites: A metabolomics-based analysis. J. Proteome Res. 2017, 16, 970-977. [CrossRef] [PubMed]

9. Davison, G.; Vinaixa, M.; McGovern, R.; Beltran, A.; Novials, A.; Correig, X.; McClean, C. Metabolomic response to acute hypoxic exercise and recovery in adult males. Front. Physiol. 2018, 9, 1682. [CrossRef] [PubMed]

10. Hodgson, A.B.; Randell, R.K.; Boon, N.; Garczarek, U.; Mela, D.J.; Jeukendrup, A.E.; Jacobs, D.M. Metabolic response to green tea extract during rest and moderate-intensity exercise. J. Nutr. Biochem. 2013, 325-334. [CrossRef] [PubMed]

11. Karl, J.P.; Margolis, L.M.; Murphy, N.E.; Carrigan, C.T.; Castellani, J.W.; Madslien, E.H.; Teien, H.-K.; Martini, S.; Montain, S.J.; Pasiakos, S.M. Military training elicits marked increases in plasma metabolomic signatures of energy metabolism, lipolysis, fatty acid oxidation, and ketogenesis. Physiol. Rep. 2017, 5, e13407. [CrossRef]

12. Lehmann, R.; Zhao, X.; Weigert, C.; Simon, P.; Fehrenbach, E.; Fritsche, J.; Machann, J.; Schick, F.; Wang, J.; Hoene, M.; et al. Medium chain acylcarnitines dominate the metabolite pattern in humans under moderate intensity exercise and support lipid oxidation. PLoS ONE 2010, 5, e11519. [CrossRef]

13. Lewis, G.D.; Farrell, L.; Wood, M.J.; Martinovic, M.; Arany, Z.; Rowe, G.C.; Souza, A.; Cheng, S.; McCabe, E.L.; Yang, E.; et al. Metabolic signatures of exercise in human plasma. Sci. Transl. Med. 2010, 2, 33-37. [CrossRef] [PubMed]

14. Nieman, D.C.; Gillitt, N.D.; Knab, A.M.; Shanely, R.A.; Pappan, K.L.; Jin, F.; Lila, M.A. Influence of a polyphenol-enriched protein powder on exercise-induced inflammation and oxidative stress in athletes: A randomized trial using a metabolomics approach. PLoS ONE 2013, 8, e72215. [CrossRef] [PubMed]

15. Al-Khelaifi, F.; Diboun, I.; Donati, F.; Botrè, F.; Alsayrafi, M.; Georgakopoulos, C.; Suhre, K.; Yousri, N.A.; Elrayess, M.A. A pilot study comparing the metabolic profiles of elite-level athletes from different sporting disciplines. Sports Med. Open 2018, 4, 2. [CrossRef] [PubMed]

16. Knab, A.M.; Nieman, D.C.; Gillitt, N.D.; Shanely, R.A.; Cialdella-Kam, L.; Henson, D.A.; Sha, W. Effects of a flavonoid-rich juice on inflammation, oxidative stress, and immunity in elite swimmers: A metabolomics-based approach. Int. J. Sport Nutr. Exerc. Metab. 2013, 23, 150-160. [CrossRef] [PubMed]

17. Manaf, F.A.; Lawler, N.; Peiffer, J.J.; Maker, G.L.; Boyce, M.C.; Fairchild, T.J.; Broadhurst, D. Characterizing the plasma metabolome during and following a maximal exercise cycling test. J. Appl. Physiol. 2018, 125, 1193-1203. [CrossRef] [PubMed] 
18. Messier, F.M.; Le Moyec, L.; Santi, C.; Gaston, A.-F.; Triba, M.N.; Roca, E.; Durand, F. The impact of moderate altitude on exercise metabolism in recreational sportsmen: A nuclear magnetic resonance metabolomic approach. Appl. Physiol. Nutr. Metab. Physiol. Appl. Nutr. Metab. 2017, 42, 1135-1141. [CrossRef]

19. Nieman, D.C.; Shanely, R.A.; Gillitt, N.D.; Pappan, K.L.; Lila, M.A. Serum metabolic signatures induced by a three-day intensified exercise period persist after $14 \mathrm{~h}$ of recovery in runners. J. Proteome Res. 2013, 12, 4577-4584. [CrossRef]

20. Nieman, D.C.; Shanely, R.A.; Luo, B.; Meaney, M.P.; Dew, D.A.; Pappan, K.L. Metabolomics approach to assessing plasma 13- and 9-hydroxy-octadecadienoic acid and linoleic acid metabolite responses to 75-km cycling. Am. J. Physiol. Regul. Integr. Comp. Physiol. 2014, 307, 68-74. [CrossRef]

21. Ra, S.-G.; Maeda, S.; Higashino, R.; Imai, T.; Miyakawa, S. Metabolomics of salivary fatigue markers in soccer players after consecutive games. Appl. Physiol. Nutr. Metab. Physiol. Appl. Nutr. Metab. 2014, 39, 1120-1126. [CrossRef]

22. Stander, Z.; Luies, L.; Mienie, L.J.; Keane, K.M.; Howatson, G.; Clifford, T.; Stevenson, E.J.; Loots, D.T. The altered human serum metabolome induced by a marathon. Metabolomics Off. J. Metabolomic Soc. 2018, 14, 150. [CrossRef] [PubMed]

23. Danaher, J.; Gerber, T.; Wellard, R.M.; Stathis, C.G.; Cooke, M.B. The use of metabolomics to monitor simultaneous changes in metabolic variables following supramaximal low volume high intensity exercise. Metabolomics 2015, 12, 7. [CrossRef]

24. Howe, C.C.F.; Alshehri, A.; Muggeridge, D.; Mullen, A.B.; Boyd, M.; Spendiff, O.; Moir, H.J.; Watson, D.G. Untargeted metabolomics profiling of an $80.5 \mathrm{~km}$ simulated treadmill ultramarathon. Metabolites 2018, 8, 14 . [CrossRef] [PubMed]

25. Neal, C.M.; Hunter, A.M.; Brennan, L.; O'Sullivan, A.; Hamilton, D.L.; De Vito, G.; Galloway, S.D.R. Six weeks of a polarized training-intensity distribution leads to greater physiological and performance adaptations than a threshold model in trained cyclists. J. Appl. Physiol. 2013, 114, 461-471. [CrossRef] [PubMed]

26. Peake, J.M.; Tan, S.J.; Markworth, J.F.; Broadbent, J.A.; Skinner, T.L.; Cameron-Smith, D. Metabolic and hormonal responses to isoenergetic high-intensity interval exercise and continuous moderate-intensity exercise. Am. J. Physiol. Endocrinol. Metab. 2014, 307, 539-552. [CrossRef] [PubMed]

27. Pechlivanis, A.; Kostidis, S.; Saraslanidis, P.; Petridou, A.; Tsalis, G.; Veselkov, K.; Mikros, E.; Mougios, V.; Theodoridis, G.A. ${ }^{1} \mathrm{H}$ NMR study on the short- and long-term impact of two training programs of sprint running on the metabolic fingerprint of human serum. J. Proteome Res. 2013, 12, 470-480. [CrossRef] [PubMed]

28. Zafeiridis, A.; Chatziioannou, A.C.; Sarivasiliou, H.; Kyparos, A.; Nikolaidis, M.G.; Vrabas, I.S.; Pechlivanis, A.; Zoumpoulakis, P.; Baskakis, C.; Dipla, K.; et al. Global metabolic stress of isoeffort continuous and high intensity interval aerobic exercise: A comparative ${ }^{1} \mathrm{H}$ NMR metabonomic study. J. Proteome Res. 2016, 15, 4452-4463. [CrossRef] [PubMed]

29. Muhsen Ali, A.; Burleigh, M.; Daskalaki, E.; Zhang, T.; Easton, C.; Watson, D.G. Metabolomic profiling of submaximal exercise at a standardized relative intensity in healthy adults. Metabolites 2016, 6, 9. [CrossRef] [PubMed]

30. Castro, A.; Duft, R.G.; Ferreira, M.L.V.; de Andrade, A.L.L.; Gáspari, A.F.; de Marchi Silva, L.; de Oliveira-Nunes, S.G.; Cavaglieri, C.R.; Ghosh, S.; Bouchard, C.; et al. Association of skeletal muscle and serum metabolites with maximum power output gains in response to continuous endurance or high-intensity interval training programs: The TIMES study—A randomized controlled trial. PLoS ONE 2019, 14, e0212115. [CrossRef]

31. Enea, C.; Seguin, F.; Petitpas-Mulliez, J.; Boildieu, N.; Boisseau, N.; Delpech, N.; Diaz, V.; Eugène, M.; Dugué, B. ${ }^{1} \mathrm{H}$ NMR-based metabolomics approach for exploring urinary metabolome modifications after acute and chronic physical exercise. Anal. Bioanal. Chem. 2010, 396, 1167-1176. [CrossRef]

32. Andersson Hall, U.; Edin, F.; Pedersen, A.; Madsen, K. Whole-body fat oxidation increases more by prior exercise than overnight fasting in elite endurance athletes. Appl. Physiol. Nutr. Metab. Physiol. Appl. Nutr. Metab. 2016, 41, 430-437. [CrossRef] [PubMed]

33. Pechlivanis, A.; Kostidis, S.; Saraslanidis, P.; Petridou, A.; Tsalis, G.; Mougios, V.; Gika, H.G.; Mikros, E.; Theodoridis, G.A. ${ }^{1} \mathrm{H}$ NMR-based metabonomic investigation of the effect of two different exercise sessions on the metabolic fingerprint of human urine. J. Proteome Res. 2010, 9, 6405-6416. [CrossRef] [PubMed] 
34. Wang, F.; Han, J.; He, Q.; Geng, Z.; Deng, Z.; Qiao, D. Applying (1)H NMR spectroscopy to detect changes in the urinary metabolite levels of Chinese half-pipe snowboarders after different exercises. J. Anal. Methods Chem. 2015, 2015, 315217. [CrossRef] [PubMed]

35. Yan, B.; A, J.; Wang, G.; Lu, H.; Huang, X.; Liu, Y.; Zha, W.; Hao, H.; Zhang, Y.; Liu, L.; et al. Metabolomic investigation into variation of endogenous metabolites in professional athletes subject to strength-endurance training. J. Appl. Physiol. Bethesda Md 1985 2009, 106, 531-538. [CrossRef] [PubMed]

36. Prado, E.; Souza, G.H.M.F.; Pegurier, M.; Vieira, C.; Lima-Neto, A.B.M.; Assis, M.; Guedes, M.I.F.; Koblitz, M.G.B.; Ferreira, M.S.L.; Macedo, A.F.; et al. Non-targeted sportomics analyses by mass spectrometry to understand exercise-induced metabolic stress in soccer players. Int. J. Mass Spectrom. 2017, 418, 1-5. [CrossRef]

37. Sun, T.; Wu, Y.; Wu, X.; Ma, H. Metabolomic profiles investigation on athletes' urine 35 minutes after an 800-meter race. J. Sports Med. Phys. Fitness 2017, 57, 839-849. [CrossRef] [PubMed]

38. Berton, R.; Conceição, M.S.; Libardi, C.A.; Canevarolo, R.R.; Gáspari, A.F.; Chacon-Mikahil, M.P.T.; Zeri, A.C.; Cavaglieri, C.R. Metabolic time-course response after resistance exercise: A metabolomics approach. J. Sports Sci. 2017, 35, 1211-1218. [CrossRef] [PubMed]

39. Valério, D.F.; Berton, R.; Conceição, M.S.; Canevarolo, R.R.; Chacon-Mikahil, M.P.T.; Cavaglieri, C.R.; Meirelles, G.V.; Zeri, A.C.; Libardi, C.A. Early metabolic response after resistance exercise with blood flow restriction in well-trained men: A metabolomics approach. Appl. Physiol. Nutr. Metab. Physiol. Appl. Nutr. Metab. 2018, 43, 240-246. [CrossRef]

40. Nieman, D.C.; Wentz, L.M. The compelling link between physical activity and the body's defense system. J. Sport Health Sci. 2019, 8, 201-217. [CrossRef]

41. Nieman, D.C.; Gillitt, N.D.; Sha, W. Identification of a select metabolite panel for measuring metabolic perturbation in response to heavy exertion. Metabolomics 2018, 14, 147. [CrossRef]

42. Nieman, D.C.; Gillitt, N.D.; Sha, W.; Esposito, D.; Ramamoorthy, S. Metabolic recovery from heavy exertion following banana compared to sugar beverage or water only ingestion: A randomized, crossover trial. PLoS ONE 2018, 13, e0194843. [CrossRef] [PubMed]

43. Nieman, D.C.; Gillitt, N.D.; Henson, D.A.; Sha, W.; Shanely, R.A.; Knab, A.M.; Cialdella-Kam, L.; Jin, F. Bananas as an energy source during exercise: A metabolomics approach. PLoS ONE 2012, 7, e37479. [CrossRef] [PubMed]

44. Sparks, L.M. Exercise training response heterogeneity: Physiological and molecular insights. Diabetologia 2017, 60, 2329-2336. [CrossRef] [PubMed]

45. Liberati, A.; Altman, D.G.; Tetzlaff, J.; Mulrow, C.; Gøtzsche, P.C.; Ioannidis, J.P.A.; Clarke, M.; Devereaux, P.J.; Kleijnen, J.; Moher, D. The PRISMA statement for reporting systematic reviews and meta-analyses of studies that evaluate health care interventions: Explanation and elaboration. PLoS Med. 2009, 6, e1000100. [CrossRef] [PubMed]

46. Fabbri, S.; Silva, C.; Hernandes, E.; Octaviano, F.; Di Thommazo, A.; Belgamo, A. Improvements in the StArt tool to better support the systematic review process. In Proceedings of the 20th International Conference on Evaluation and Assessment in Software Engineering, Limerick, Ireland, 1-3 June 2016; ACM: New York, NY, USA, 2016.

47. Heaney, L.M.; Deighton, K.; Suzuki, T. Non-targeted metabolomics in sport and exercise science. J. Sports Sci. 2017, 37, 959-967. [CrossRef] [PubMed]

(C) 2019 by the authors. Licensee MDPI, Basel, Switzerland. This article is an open access article distributed under the terms and conditions of the Creative Commons Attribution (CC BY) license (http://creativecommons.org/licenses/by/4.0/). 